\title{
Direct replications in the era of open sampling
}

\author{
Commentary on "Making Replication Mainstream" by Zwaan, Etz, Lucas, and Donnellan \\ To appear in Behavioral and Brain Sciences
}

\author{
Gabriele Paolacci \\ Rotterdam School of Management, Erasmus University Rotterdam \\ gpaolacci@rsm.nl \\ Jesse Chandler \\ Mathematica Policy Research \\ Institute for Social Research, University of Michigan \\ jjchandl@umich.edu
}

\begin{abstract}
Data collection in psychology increasingly relies on "open populations" of participants recruited online, which presents both opportunities and challenges for replication. Reduced costs and the possibility to access the same populations allows for more informative replications. However, researchers should ensure the directness of their replications by dealing with the threats of participant nonnaiveté and selection effects.
\end{abstract}


When the "crisis of confidence" struck psychology, giving a new pace to the academic debate on replications, a parallel revolution was happening in the field: Data collection rapidly moved away from near exclusive dependence on traditional participant pools (e.g., undergraduate samples) and towards sampling from online marketplaces where adults complete tasks (e.g., academic surveys) in exchange for compensation. About five years later, virtually any major journal in psychology and beyond routinely publishes studies conducted on Amazon Mechanical Turk, Prolific, or other third-party platforms (Stewart, Chandler, and Paolacci 2017; Chandler \& Shapiro 2016). Importantly, these marketplaces are typically "open" on both ends: Compared to any traditional participant pool (e.g., psychology undergraduates in a Midwestern university), few restrictions exist about who can join the participant pool and who can recruit participants from these populations.

Zwaan, Etz, Lucas, and Donnellan (ZELD) provide a compelling case for direct replications, emphasizing both the necessity of being able to reproduce the procedures used in the original experiments and the lack of structural obstacles to make replications a habit in the field. However, ZELD do not discuss how direct replications are affected by the current practices of data collection, and in particular by researchers' increasing reliance on open sampling. We build on the target article by highlighting how open sampling presents opportunities to make direct replication mainstream and the challenges of conducting a proper direct replication using these samples.

Open sampling can remove barriers to making replications habitual, while also making attempted replications more conclusive. First, data collection from open populations is comparatively faster and cheaper (even controlling for pay rate, Goodman \& Paolacci 2017). This reduces concerns about committing scarce resources to replication, and allows recruiting larger samples given the same time and budget allocated to conducting a replication. This is beneficial for any study and particularly for replication studies, that demand even more participants than original studies to make conclusive statements (Simonsohn 2015).

Second, original studies conducted on open populations can be replicated by different researchers using the same population. Sharing the same population does not make replications perfect, and we discuss below how this is also true of open populations; however, a shared population is a necessary precondition for more informative failed replications. Samples from different sources vary substantially on many characteristics (Kennedy et al., 2016). All else being equal, a failed replication on the same population is both less suggestive of hidden moderators and less ambiguous about which hidden moderators might be at play. When the replicator's goal is to increase the directness of a replication, rather than discovering population-level moderators of the target effect, open populations further reduce the "Context Is Too Variable" concern that ZELD address in the target article.

Despite these advantages, open sampling only increases the directness of a replication if researchers pay appropriate attention to the sampling methodology. First, despite intuitions of the contrary, open populations have a large but limited number of participants (Difallah, Filatova, and Ipeirotis 2018, Stewart et al. 2015). Combined with researchers using these populations to 
conduct many studies that are often high-powered, this has resulted in concerns about participant nonnaivete that are relevant to replication. Open populations include many participants who are experienced with research participation, and who become more experienced over time with specific research paradigms and instruments. Illustratively, popular paradigms are known to a large majority of participants (e.g., Chandler, Mueller, and Paolacci 2014, Thomson \& Oppenheimer 2016). ZELD highlight how some findings in cognitive psychology (i.e., perception/action, memory, and language) replicate even with participants who were previously exposed to them (Zwaan et al. 2017). However, this is not necessarily the case for any paradigm, and may be particularly not true of replications in other psychological fields. There is evidence that experimental manipulations in social psychology and decision-making that convey experience (e.g., tasks conducted under time pressure) or factual knowledge (e.g., numeric estimates following different numeric anchors) become less strong with repeated exposure. This can result in replications that are less statistically powerful than intended (Chandler et al. 2015, Devoe \& House 2016, Rand et al. 2014), and nonnaive participants should therefore be accounted for by direct replicators (Chander et al. 2014).

Second, samples obtained from open populations are not probability samples, and thus can still vary due to procedural differences in sampling. Participants of open populations selfselect into studies by choosing from many that differ across observable characteristics (e.g., payment, task description) that may make them more or less attractive to different people. Researchers may place explicit constraints on participant eligibility that have a measurable impact on data quality (e.g., worker reputation scores; Peer, Vosgerau \& Acquisti, 2014 or nationality; Chandler \& Shapiro, 2016) but may not be reported. Other recruitment criteria that are not deliberately selected may still be impactful. The diversity of open populations compounds this concern, because it suggests a comparatively high potential for procedural differences to meaningfully affect sample composition. Though evidence is still scarce, researchers have found that sample demographics fluctuate with time-of-the-day and day-of-the week (Casey et al. 2017, Arechar, Kraft-Todd, and Rand 2017). This implies the need for direct replicators to consider aspects of the original design (e.g., timing, study compensation) that are not typically assumed to be hidden moderators in undergraduate samples that are less diverse and less characterized by self-selection. It also emphasizes that, in the era of open samples, original authors are as responsible as direct replicators to support replicability efforts by reporting their sampling choices in sufficient detail to ensure meaningful replication.

In sum, we applaud the target article on convincingly addressing the most commonly raised concerns about replication, and put some of the target article's insights within the context of today's dominant practice in data collection - open sampling. We hope this commentary will contribute to make informative replication mainstream, by encouraging researchers to both embrace the advantages of open sampling and consider what transparent reporting of methods and direct replication means when using these samples. 


\section{References}

Arechar, A. A., Kraft-Todd, G. T., \& Rand, D. G. (2017). Turking overtime: how participant characteristics and behavior vary over time and day on Amazon Mechanical Turk. Journal of the Economic Science Association, 3(1), 1-11.

Casey, L., Chandler, J., Levine, A. S., Proctor, A., \& Strolovitch, D. Z. (2017). Intertemporal differences among MTurk worker demographics. SAGE Open.

Chandler, J., Mueller, P., \& Paolacci, G. (2014). Nonnaïveté among Amazon Mechanical Turk workers: Consequences and solutions for behavioral researchers. Behavior Research Methods, 46(1), 112-130.

Chandler, J., \& Shapiro, D. (2016). Conducting clinical research using crowdsourced convenience samples. Annual Review of Clinical Psychology, 12, 53-81.

Chandler, J., Paolacci, G., Peer, E., Mueller, P., \& Ratliff, K. A. (2015). Using nonnaive participants can reduce effect sizes. Psychological Science, 26(7), 1131-1139.

Difallah, D., Filatova, E., \& Ipeirotis, P. (2018). Demographics and Dynamics of Mechanical Turk Workers. In Proceedings of WSDM 2018: The Eleventh ACM International Conference on Web Search and Data Mining, Marina Del Rey, CA, USA, February 5-9, 2018 (WSDM 2018), 9

Goodman, J. K., \& Paolacci, G. (2017). Crowdsourcing consumer research. Journal of Consumer Research, 44(1), 196-210.

Kennedy, C., Mercer, A., Keeter, S., Hatley, N., Mcgeeney, K., \& Gimenez, A. (2016). Evaluating online nonprobability surveys. Pew Research Center. Available at: http://www. pewresearch. org/2016/05/02/evaluating-online-nonprobability-surveys/(accessed September 2016).

Peer, E., Vosgerau, J., \& Acquisti, A. (2014). Reputation as a sufficient condition for data quality on Amazon Mechanical Turk. Behavior research methods, 46(4), 1023-1031.

Rand, D. G., Peysakhovich, A., Kraft-Todd, G. T., Newman, G. E., Wurzbacher, O., Nowak, M. A., \& Greene, J. D. (2014). Social heuristics shape intuitive cooperation. Nature Communications, 5, 3677.

Simonsohn, U. (2015). Small Telescopes: Detectability and the Evaluation of Replication Results. Psychological Science, 26, 559-569.

Stewart, N., Chandler, J., \& Paolacci, G. (2017). Crowdsourcing Samples in Cognitive Science. Trends in Cognitive Sciences, 21(10), 736-748.

Thomson, K. S., \& Oppenheimer, D. M. (2016). Investigating an alternate form of the cognitive reflection test. Judgment and Decision Making, 11(1), 99-113.

Zwaan, R. A., Pecher, D., Paolacci, G., Bouwmeester, S., Verkoeijen, P., Dijkstra, K., \& Zeelenberg, R. (2017). Participant nonnaiveté and the reproducibility of cognitive psychology. Psychonomic Bulletin \& Review, 1-5. 